\title{
Scaling studies of the valence transition in cerium-based alloys $(*)$
}

\author{
M. A. Manheimer and R. D. Parks \\ Department of Physics and Astronomy \\ University of Rochester, Rochester, New York 14627, U.S.A.
}

\begin{abstract}
Résumé. - Les paramètres de l'équation d'état de Landau, qui décrit la transition de valence dans les alliages de cérium, ont été mesurés pour un certain nombre d'additifs de différents rayons atomiques et de différentes valences. Ceci nous a permis de déterminer l'importance relative de l'interaction coulombienne et des effets associés au réseau cristallin sur la transition de valence.

Abstract. - The parameters of the Landau equation of state, which describes the valence transition in Ce-based alloys, have been measured for a number of additives of varying atomic radii and valence. This in turn has allowed us to isolate the coulombic and lattice effects on the valence instability.
\end{abstract}

1. Introduction. - The isomorphic valence transition in $\mathrm{Ce}_{1-x} \mathrm{Th}_{x}$ has been well characterized in a series of studies $[1,2]$, which both established the mean field nature of the transition in the vicinity of the critical point $\left(x_{0}=0.265, T_{0}=148 \mathrm{~K}\right)$ and determined a quantitative Landau equation of state which is valid in a large sector of $x-T$ space encompassing the critical point. The purpose of extending this work to include additives other than $T h$ was to gain information concerning the driving mechanism(s) for the valence transition. A preliminary report of the work presented here appears in reference [3].

2. Experimental results. - The alloy systems studied were $\mathrm{Ce}_{0.9-x} \mathrm{X}_{x} \mathrm{Th}_{0.1}$ with $\mathrm{X}=\mathrm{Th}, \mathrm{Sc}, \mathrm{La}$, $\mathrm{Eu}, \mathrm{Gd}, \mathrm{Dy}, \mathrm{Er}, \mathrm{Yb}$, and Lu. The $10 \% \mathrm{Th}$ was present in all samples to suppress the formation of hexagonal $\beta$-phase Cerium. Since it was established previously $[1,2]$ that the electrical resistivity couples linearly to the order parameter in Ce-based alloys in the vicinity of the critical point, it was this (relatively simple to measure) property which was used exclusively in the present study to determine the critical parameters.

In all of the ternary systems studied, a linear dependence of the first-order transition temperature $T_{\mathrm{c}}$ on concentration $x$ was observed in the entire concentration range $0 \leqslant x \leqslant x_{0}, x_{0}$ being the critical concentration (see, e.g., figure 1 of reference [3]). Moreover, for the trivalent additives, $\mathrm{d} T_{\mathrm{c}} / \mathrm{d} x$ depends linearly on size of the additive as seen in figure 1 . Fitting the data for the trivalent additives to a straight line and assuming an arbitrary dependence $f(N)$

$\left(^{*}\right)$ Work supported in part by the Army Research Office and the Office of Naval Research.

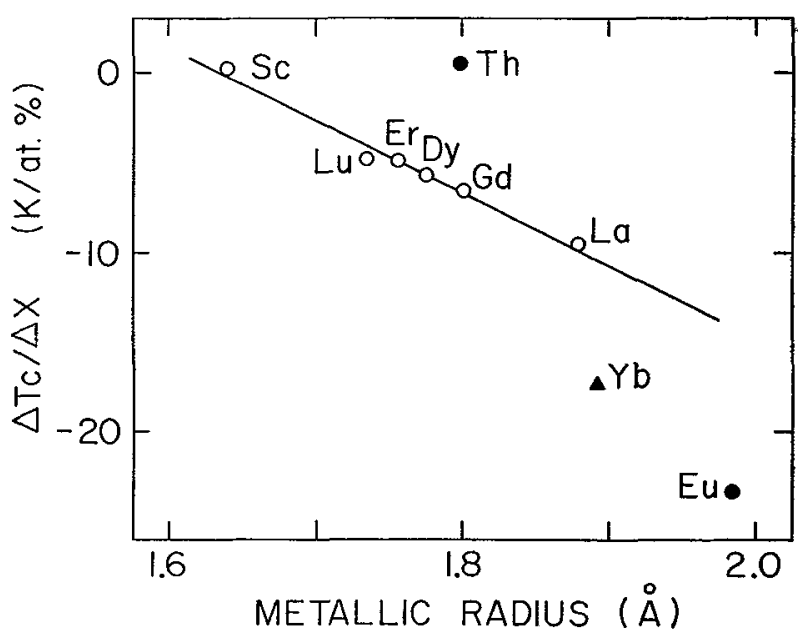

Fig. 1. - Slope of coexistence curve $\Delta T_{\mathrm{c}} / \Delta x$ versus metallic radius of different additives $\mathrm{X}$ in $\mathrm{Ce}_{0.9-x} \mathrm{X}_{x} \mathrm{Th}_{0.1}$.

of $\mathrm{d} T_{\mathrm{c}} / \mathrm{d} x$ on the valence $N$ of the ternary additive results in

$$
\frac{\mathrm{d} T_{\mathrm{c}}}{\mathrm{d} x}=-70 \frac{R-R_{0}}{R_{\mathrm{o}}}+f(N)
$$

where $R_{0}=1.759 \AA$, to be identified below with the metallic radius of $\mathrm{Ce}$ at the critical point. Plotting $F(N)=\mathrm{d} T_{\mathrm{c}} \mathrm{d} x+70\left(R-R_{0}\right) / R_{0}$ versus $N$ (see figure 2) with $N=4$ for Th, $N=2$ for $\mathrm{Eu}$, and where we have placed the $\mathrm{Yb}$ data point on the resulting straight line, corresponding to $N=2.2$ for $\mathrm{Yb}$ (discussed below), yields to a good approximation a linear dependence of $f(N)$ on $N$ and allows us to re-express eq. (1) as

$$
\frac{\mathrm{d} T_{\mathrm{c}}}{\mathrm{d} x}=-70 \frac{R-R_{0}}{R_{0}}+30 \frac{N-N_{0}}{N_{0}}
$$




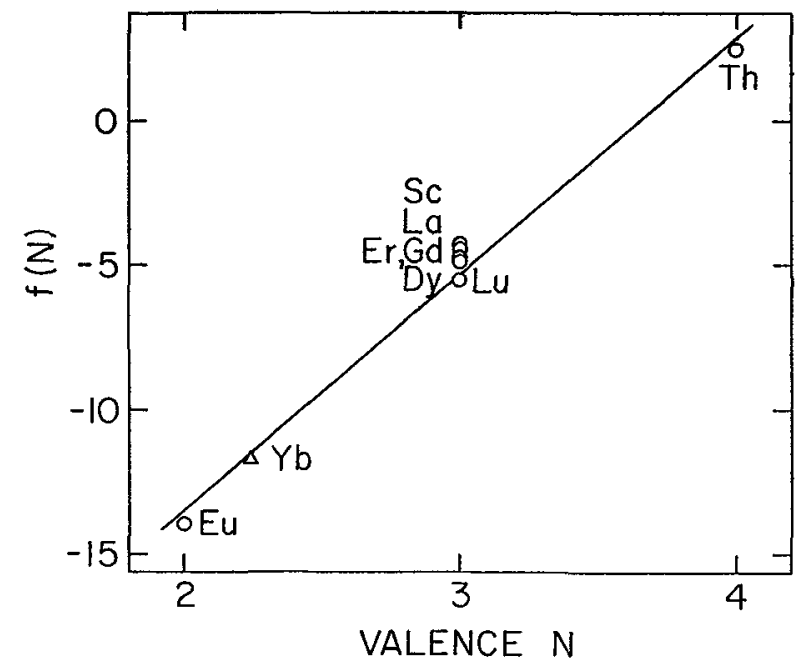

Fig. 2. - Plot of $\rho(N)$ (see text) versus valence $N$ for different additives.

with $N_{0}=3.66$, corresponding empirically, and as we shall discuss below, to the valence of $\mathrm{Ce}$ at critical point.

A second objective of the ternary alloy study was to examine the scaling properties of other terms in the Landau equation of state with different ternary additives (hence, different chemical fields). The equation of state as determined from the early studies [2] of $\mathrm{Ce}_{1-x} \mathrm{Th}_{x}$ can be expressed as

$$
\Delta T=a(\Delta \rho)^{3}+b(\Delta x)(\Delta \rho)+c \Delta x
$$

where the dimensionless quantities $\Delta T, \Delta x$ and $\Delta \rho$ (resistivity) are all scaled to their critical values, e.g., $\Delta T=\left(T-T_{0}\right) / T_{0}$. The behavior of

$$
c \equiv x_{0} / T_{0}\left(\Delta T_{\mathrm{c}} / \Delta x\right)
$$

is expressed in Eq. (2). The values for $a$ and $b$ are shown in figure 3 . To within experimental uncertainty $a$ is independent of both $R$ and $N$; having the value $a=0.52$. On the other hand the parameter $b$ is independent of $N$, but depends strongly (and linearly) on $R$. The radius chosen for $\mathrm{Yb}$ again corresponds

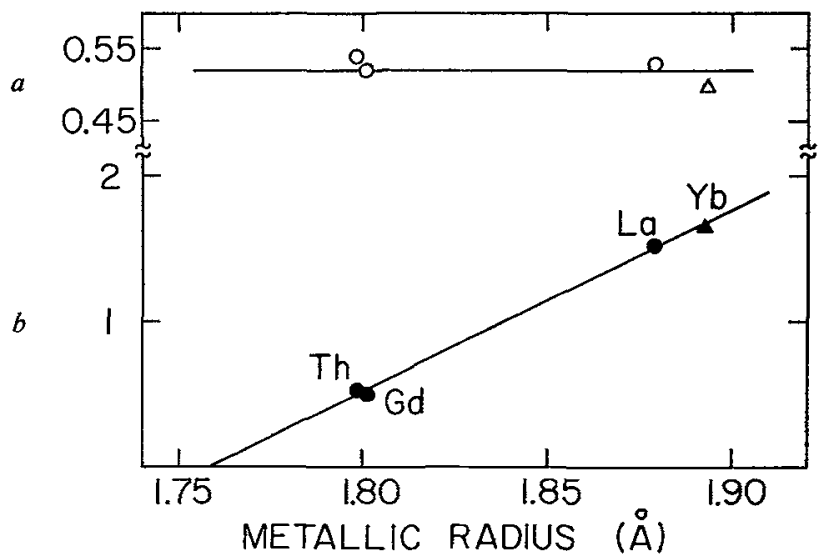

Fig. 3. - Dependence of equation of state parameters $a$ and $b$ on metallic radius of additive. to a valence of 2.2 , hence we have a corroborative check on that choice of valence. Extrapolation of $b(R)$ to $b=0$ provides the motivation for choosing the fiducial radius $R_{0}=1.759 \AA$; moreover, this value is close to the value $R_{\mathrm{m}}=1.751 \AA$ for the radius of $\mathrm{Ce}$ at the midpoint of the pressure-induced first order phase transition in $\mathrm{Ce}$ at room temperature (see reference [4] and references therein).

3. Discussion. - In the above we have identified the fiducial values $R_{0}=1.759 \AA$ and $N_{0}=3.66$ with the metallic radius and valence of $\mathrm{Ce}$ at the critical point. The finding, $N_{0}=3.66$, is a particularly significant result of the present study, since it differs considerably from the commonly accepted estimate, $N_{0}=3.26$, made by Gschneidner and Smoluchowski (GS) [4] for pure Cerium, based upon X-ray diffraction and other studies. We believe that the estimate of GS is low because of their assumption that the valence depends linearly on lattice constant in the complete valence range $3.0 \leqslant N \leqslant 4.0$. Such a linear dependence has been observed $[1,2]$ in the immediate vicinity of $T_{0}$, but there is no rationale for assuming it to hold over a large range of either $N$ or $R$. We estimate the valence for either pure $\mathrm{Ce}$ or Ce in the ternary systems studied here to fall in the range 3.8-4.0 deep in the mixed valence phase, e.g., $T \rightarrow 0$ or $P \gg P_{0}, P_{0}$ being the transforming pressure.

Summarizing the salient results of the equation of state scaling studies, we note that the 3rd term on the RHS of Eq. (4), which expresses a renormalization of $T_{0}$ and is not a bootstrapping term, depends equally strongly on the valence and metallic radius of the ternary additive. We believe as discussed previously [3] that the dominant size effect is to simulate either positive or negative pressure thereby moving the $4 \mathrm{f}$ level with respect to the Fermi level, and the dominant valence effect is basically a coulombic effect, which can both alter the width of the if level and change its energy with respect to the Fermi level. Since the (bootstrapping) second term on the RHS of Eq. (3) depends on the size but not the valence of the additive, we look to lattice effects as the source of the driving mechanism for the valence transition. Among lattice related effects, it would seem to be difficult to choose between band deformation effects induced by lattice deformations and acoustic strain effects as relevant to the problem of phase separation in alloys. A more complete account of the work reported here will be published elsewhere.

\section{References}

[1] LaWrence, J. M., Crofot, M. C. and Parks, R. D., Phys, Rev. Lett. 35 (1975) 289.

[2] Parks, R. D. and LaWrence, J. M., AIP Conf. Proc. 29 (1976) 429.

[3] Manheimer, M. A. and Parks, R. D., Valence Instabilities and Related Narrow Band Phenomena, edited by R. D. Parks (Plenum, New York) 1977, p. 447.

[4] Gschneidner, K. A., Jr and SMoluchowski, R., J. LessCommon Metals 5 (1963) 374. 